\title{
Mucormycosis of maxilla
}

\author{
Priyanka Choudhary B ${ }^{1}$, Deepak Bhargava², \\ Vidyadevi Chandavarkar S33, Ritika Sharma ${ }^{4}$
}

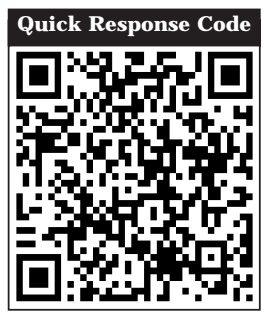

doi : 10.5866/2014.611503

1Postgraduate Student

${ }^{2}$ Professor \& HOD

${ }^{3}$ Senior Lecturer

${ }^{4}$ Lecturer

Department of Oral Pathology \& Microbiology School of Dental Sciences, Sharda University, Greater Noida

\section{Article Info:}

Received: October 8, 2013

Review Completed: November 7, 2013

Accepted: December 10, 2013

Available Online: February, 2014 (www.nacd.in)

(C) NAD, 2014 - All rights reserved

Email for correspondence:

priyankachoudhary.dr@gmail.com

\section{ABSTRACT:}

Mucormycosis (al so called zygomycosis) is a rare fungal infection caused by organisms that belong to a group of fungi called mucormycotina. The infection is more common among people with weakened immune system, although it can occur (rarely) in people who are otherwise healthy. Rapid diagnosis and initiation of therapy is critical due to acute fulminant nature of

infection. I nvasive mucormycosis is characterized by rapid development of tissue necrosis as a result of vascular invasion and subsequent thrombosis. In addition to host factors that predispose patients to mucormycosis, Mucorales possess virulence factors that enable the organism to cause disease. One such trait is the ability to acquire iron from the host. Depending upon the route of exposure, the infection can be pulmonary or cutaneous. In the cutaneous form, the fungus can enter skin through cuts, scraps, puncture wounds or other form of trauma to skin. The rich vascularity of maxillofacial areas usually prevents fungal infection although more virulent fungi such as those responsible for mucormycosis can often overcome this difficulty. In most cases, the infection is relentlessly progressive and results in death unless treatment with a combination of surgical debridement and antifungal therapy is initiated promptly. We hereby, present a rare case of mucormycosis of maxilla in a female patient who had history of tooth extraction.

Key words: Mucormycosis, maxillary necrosis, fungal infection

\section{Introduction}

Mucormycosis is caused by fungus of the order Mucorales and is one of the most rapidly fatal fungal infections known. ${ }^{1}$ It is the third most common fungal infection after candidiasis and aspergillosis. ${ }^{2}$ It appears as nonseptate or scarcely septate hyphae with obtuse ramifications. ${ }^{3}$ These infections are opportunistic they occur when organisms to which we are frequently exposed gain entry into the body due to decreased host defenses or through an invasive portal, such as dental extraction. ${ }^{4}$ Nose is usually the portal of entry, however perforation of skin or mucous membrane may allow entrance of the organism. ${ }^{3}$ Usually mucormycosis presents as an acute infection and manifests as rhinocerebral, pulmonary, gastrointestinal, cutaneous or disseminated form. ${ }^{5}$ The diagnosis of mucormycosis is rarely suspected and antemortem diagnosis is made 
in only $23-50 \%$ cases. ${ }^{2}$ We report a rare case of mucormycosis of maxilla in an immunocompetant patient after tooth extraction.

\section{Case report}

A 48-year old female patient reported to the department with the chief complaint of pain in the left upper jaw accompanied by hearing and nose blockage on same side since one month. The pain was moderate and continuous in nature which subsided on taking medication. The patient had undergone extraction of maxillary left third molar one month back. Following extraction, the socket never healed completely and there was spread of infection to the left temporal and infratemporal region. On examination her vitals were normal.

I ntraoral examination reveal ed exposed necrotic bone from maxillary left lateral incisor till the third molar region extending buccally and palatally (Figure 1). Based on history and clinical examination differential diagnosis of Candidiasis, Actinomycosis, Aspergillosis and Mucormycosis were reached.

On radiographic examination, paranasal sinus view showed haziness of left maxillary sinus (Figure 2). Computerized tomography revealed deviated nasal septum and sinus filled with inflammatory lining (Figure 3). Orthopantomogram revealed radiolucency indicating unhealed socket with respect to 28 . Routine hematological investigations revealed hemoglobin of $10.30 \mathrm{gm} \%$, packed cell volume of $34.10 \%$, mean corpuscular volume of $77.90 \mathrm{fl}, \mathrm{MCH}$ of $23.50 \mathrm{pg}$. KFT revealed uric acid of $6.50 \mathrm{mg} / \mathrm{dl}$. Blood sugar levels were normal. Patient was HIV and Hepatitis B negative.

The microbiology culture report reveal ed cotton wooly appearance colonies which was suggestive of fungal infection (Figure 4). An incisional biopsy was done. Histopathological examination of $\mathrm{H} \& \mathrm{E}$ stained section revealed necrosed vacoulated connective tissue stroma with tags of overlying stratified squamous epithelium. Numerous fungi in the form of spores and refractile nonseptate hyphae were seen (Figures 5). PAS stain further identified ribbon like, non-septate hyphae along with developing spores (Figures 6). Based on histopathology, final diagnosis of mucormycosis of maxilla was made and patient was appropriately managed.

\section{Discussion}

Organisms of the Class Zygomycetes were first documented by Ribes et al in humans in 1800 . However first description of Zygomycosis was reported by Platauff in $1885 .{ }^{6}$ Mucormycosis infection is associated with high mortality rates. Most human infections result from inhalation of fungal sporangio spores that have been released in air or direct inoculation of organisms into disrupted skin or mucosa. ${ }^{7}$ This microbe may be cultured from the oral cavity, nasal passages, throat and stool of healthy patients without clinical signs of infection. ${ }^{8}$

In developed countries, the disease remains uncommon and is seen mostly in patients with diabetes mellitus and hematological malignancies (HMs) undergoing chemotherapy or those who have received allogeneic stem cell transplants. In contrast, in devel oping countries, especially in I ndia, mucormycosis cases, although sporadic, occur mainly in patients with uncontrolled diabetes or trauma. ${ }^{7}$

Hallmark of mucormycosis is vascular invasion resulting in thrombosis and tissue infarction/ necrosis. In most cases, the infection is relentlessly progressive and results in death unless treatment with a combination of surgical debridement and antifungal therapy is initiated. ${ }^{9}$ Depending on the extent of the infection, cutaneous mucormycosis is classified as localized when it affects only the skin or subcutaneous tissue; deep extension when it invades muscle, tendons, or bone; and disseminated when it involves other noncontiguous organs. ${ }^{7}$

The clinical signs and symptoms of mucormycosis are nonspecific. ${ }^{10}$ Patients with rhinocerebral mucormycosis usually present with malaise, headache, facial pain, swelling and low grade fever. The disease usually begins in the nasal mucosa or palate and extends in to the paranasal sinuses spreading through the surrounding vessels. 


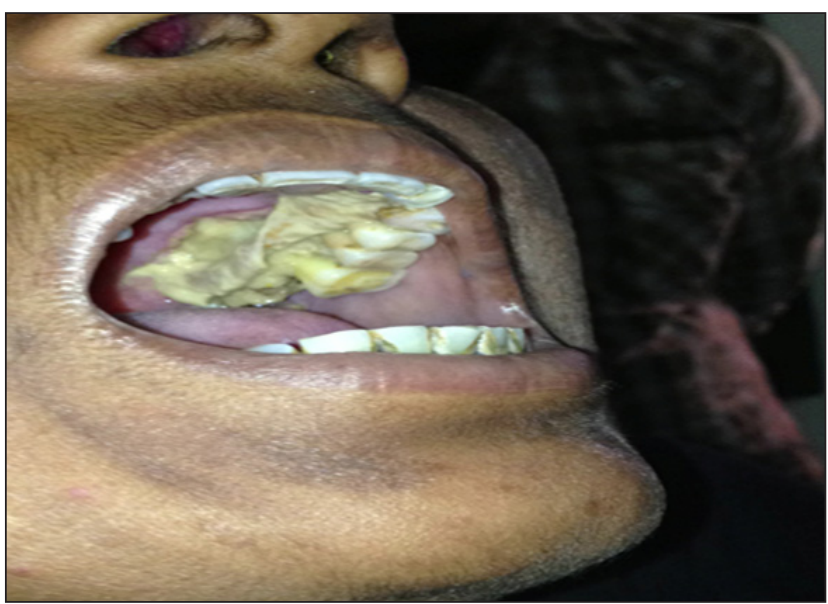

Figure 1: Exposed necrotic bone from maxillary left lateral incisor till the third molar region extending buccally and palatally.

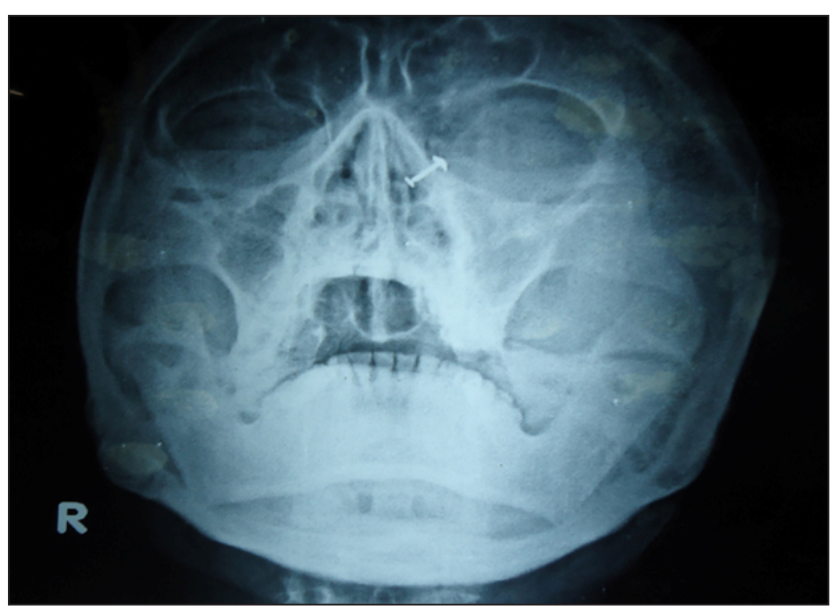

Figure 2: Paranasal sinus view shows haziness of left maxillary sinus.

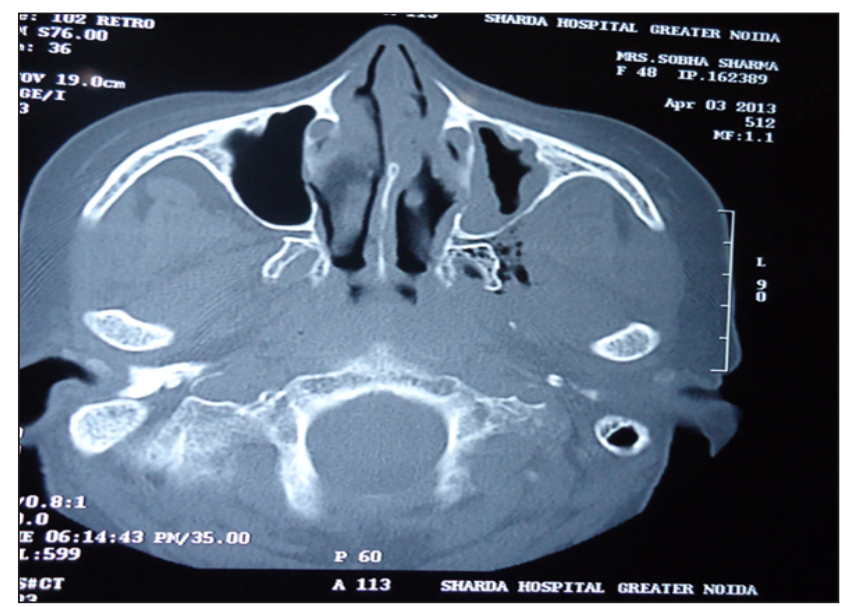

Figure 3: Computerized tomography showing deviated nasal septum and sinus filled with inflammatory lining.

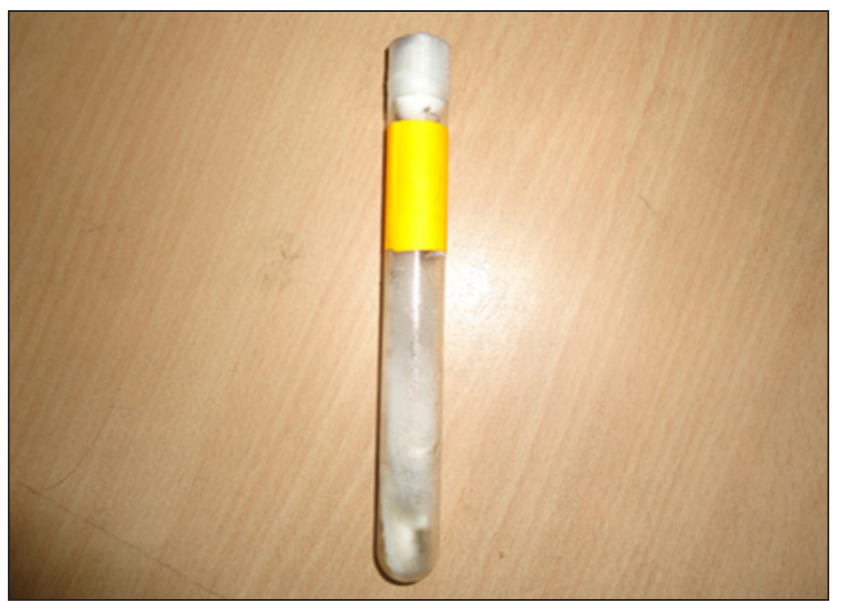

Figure 4: The microbiology culture revealing cotton wooly appearance colonies.

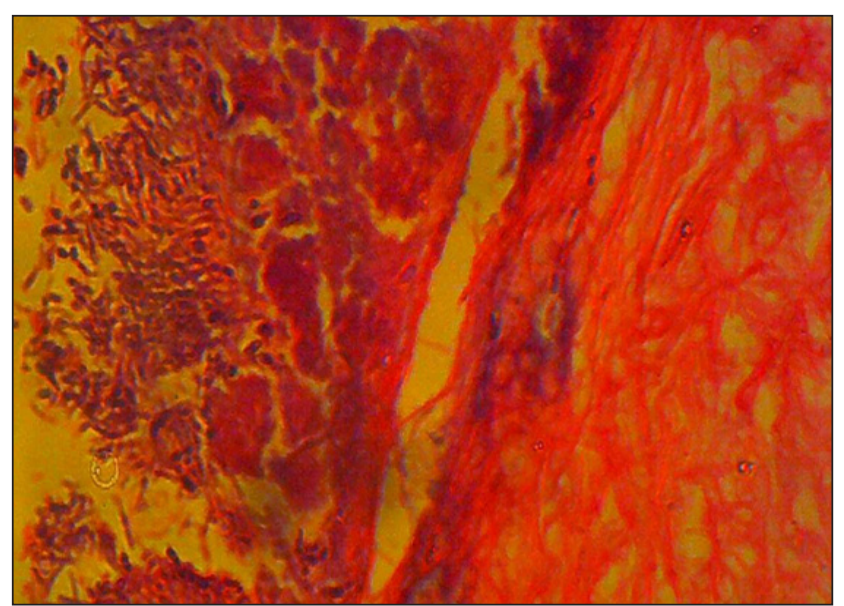

Figure 5: Numerous fungi in form of spores and refractile nonseptate hyphae( H \& E X 400)

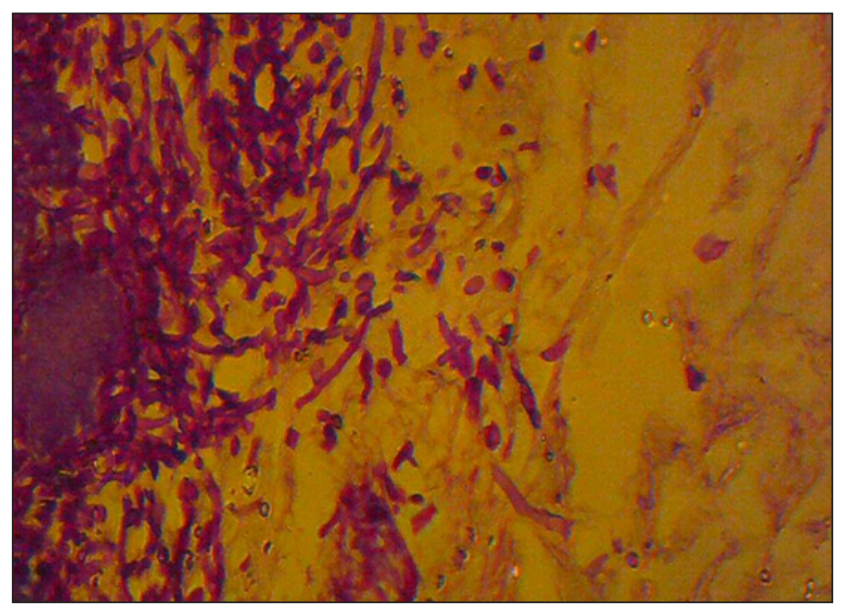

Figure 6: Non-septate hyphae al ong with developing spores(PAS X 400). 
An area of ulceration or an extraction socket in the mouth can be a port of entry for mucormycosis into the maxillofacial region, particularly when the patient is immunocompromised. ${ }^{11}$ The maxilla rarely undergoes necrosis due to its rich vascularity. Maxillary necrosis can occur due to bacterial infections such as osteomyelitis, viral infections such as herpes zoster or fungal infections such as mucormycosis, aspergillosis. Other causes other than infections include trauma, radiation, use of I ong-term corticosteroids, disorders of lipid metabolism such as Gaucher's disease. ${ }^{10}$

Radiograph of sinuses and orbits may demonstrate sinus mucosal thickening, sinus opacification without fluid levels and spotty destruction of the bony walls of the paranasal sinuses. CT scan or MRI may demonstrate erosion of bone or sinuses and helps delineate the extent of the disease. ${ }^{6} \mathrm{~A}$ variety of stains, including $\mathrm{H} \& \mathrm{E}$, Grocott-Gomori methenamine-silver nitrate, and PAS frequently reveal characteristic hyphal elements in tissue. Histopathol ogy of infected tissue often shows characteristic broad, ribbon like, thinwalled, primarily aseptate hyphae that have irregular diameters; with nondichotomous irregular branching and accompanying tissue necrosis and fungal angioinvasion. Fluorescent stains such as Calcofluour White/Blankofluor may be more revealing with small numbers of hyphae or limited tissue samples. Paradoxically, even when fungal hyphae are seen in histopathologic analysis, fungal cultures are only positive in $50 \%$ of cases because of the friable nature of nonseptated hyphae, which are frequently damaged during tissue manipulation. ${ }^{12}$

Successful management of mucormycosis includes both medical and surgical modalities. The initial medical approach to mucormycosis consists of aggressive treatment of the underlying predisposing medical condition and use of systemic antifungal agents. ${ }^{11}$

\section{Conclusion}

Mucormycosis may pose a diagnostic and therapeutic dilemma for those who are not familiar with these clinical presentations. It was regarded as a fatal infection with poor prognosis. It is rarely suspected and antemortem diagnosis is made in only $23-50 \%$ of cases. Thus it is very important to diagnose these fatal fungal infections at an early stage to reduce morbidity and mortality rates.

\section{References}

1. Bany-yaseen BY, Maita J , Mneizel T. Mucormycosis of the hard palate. J ordanian Royal Medical Services. 2006; 13: 61-63.

2. Karanth $\mathrm{M}$, Taniere $\mathrm{P}, \mathrm{Barraclough} \mathrm{J}$, Murray J A. A rare presentation of zygomycosis (mucormycosis) and review of the literature. J Clin Pathol. 2005; 58: 879-881.

3. Reddy US, Aarti R. Mucormycosis: A Case Report. J Pharm Biomed Sci. 2013; 30: 998-1001.

4. Fogarty C, Regennitter F, Viozzi CF. Invasive fungal infection of the maxilla following dental extractions in a patient with chronic obstructive pulmonary disease. J Can Dent Assoc. 2006; 72: 149-152.

5. Auluck A. Maxillary necrosis by mucormycosis. A case report and literature view. Med Oral Patol Oral Cir Bucal. 2007; 12: $360-364$

6. Thimmarasa VB, Devi P, Mehrotra V, Gupta M. Mucormycosis of the palate. J AFMC Bangladesh. 2010; 6:2931.

7. Petrikkos G, Skiada A, Lortholary O, Roilides E, Walsh TJ , Kontoyiannis DP. E pidemiology and Clinical Manifestations of Mucormycosis. Clin Infect Dis. 2012; 54: 23-34.

8. Madan R, Barde D, Rawwlani S, Chandak R. Maxillary necrosis by mucormycosis. J MGIMS. 2013; 18:67-70.

9. Spell berg B, Edwards J , I brahim A. Novel perspectives on Mucormycosis: management pathophysiology, presentation, and management. Clin Microbiol Rev. 2005; 18: 556-569.

10. Kumar J A, Babu P, Prabu K, Kumar P. Mucormycosis in maxilla: Rehabilitation of facial defects interim using removable prostheses: A clinical case report. J Pharm Bioallied Sci 2013; 5: 163-165.

11. Bakathir AA. Mucormycosis of the jaw after dental extractions: Two case reports. Sultan Qaboos University Medical J ournal. 2006; 6: 77-81.

12. Dimitrios $\mathrm{P}$, Kontoyiannis, Lewis RE. How I treat mucormycosis. Blood. 2011; 118:1216-1224. 